MELANOMA

\title{
Germline control of anti-tumour immunity
}

Germline variants of the gene encoding the secreted glycoprotein APOE are known to be risk modifiers of inflammation-associated diseases, with the most prominent example being Alzheimer disease. Among the three most common genetic variants, APOE2, APOE3 and APOE4 (the products of which differ by only one amino acid), APOE4 is a strong risk factor for Alzheimer disease, whereas APOE2 is protective. Ostendorf et al. now show that germline variants of $A P O E$ play a role in melanoma that is opposite to that played in Alzheimer disease.

Previously, the authors have shown that APOE expression is mechanistically involved in reducing melanoma progression and metastasis and affects tumour immunity by modulating myeloid cell populations. Interested in the effect of germline variants of $A P O E$, the authors analysed the growth of syngeneic YUMM1.7 tumours in mice in which the Apoe locus had been replaced with human $A P O E$ variants. This showed that tumour growth in APOE4 mice was significantly lower than in $A P O E 3$ mice, whereas tumour growth in APOE2 mice was significantly higher than in APOE3 mice. (The authors decided to compare APOE4 mice with $A P O E 2$ mice from hereon.) Flow cytometry analysis revealed that tumours in APOE4 mice had higher levels of $\mathrm{CD}^{4} 5^{+}$leukocyte infiltration, higher proportions of natural killer (NK) cells and CD8 ${ }^{+} \mathrm{T}$ cells, as well as lower proportions of myeloid-derived suppressor cells (MDSCs) and tumour-associated macrophages than tumours in APOE2 mice. After the bone marrow from $A P O E 4$ mice was transplanted into lethally irradiated Apoe wild-type mice, tumour growth in these mice was slower than in mice receiving the transplant from $A P O E 2$ mice, showing that the APOE4 variant controlled tumour growth through the haematopoietic cell compartment.

Moving to investigate the role of $A P O E$ variants in patients with melanoma, using data from the TCGA skin cutaneous melanoma study, the authors found that $A P O E$ carrier status was not associated with increased melanoma incidence, but with survival of patients with high risk of melanoma-associated death. As such, patients carrying the APOE4 variant showed improved survival outcomes relative to those carrying the APOE2 variant. These associations in patients with highrisk melanoma-associated death were confirmed using data from an independent, melanoma genomewide association study. Of note, the APOE2 variant was associated, though not significantly, with mproved survival in patients with low risk of melanoma-associated death, which is in line with its association with enhanced lifespan in the general population.

Back to the in vivo model, the authors performed single-cell RNA sequencing of $\mathrm{CD} 45^{+}$leukocytes in tumours grown in APOE4 or APOE2 mice, which showed transcriptional signatures of activation and expansion of $\mathrm{NK}$ and $\mathrm{CD} 8^{+} \mathrm{T}$ cells, with pathways of anti-tumour immunity being enriched in tumours grown in APOE4 mice compared with APOE2 mice. In line with the APOE4 variant promoting anti-tumour immunity, the depletion of $\mathrm{T}$ cells led to enhanced tumour growth in APOE4 mice to

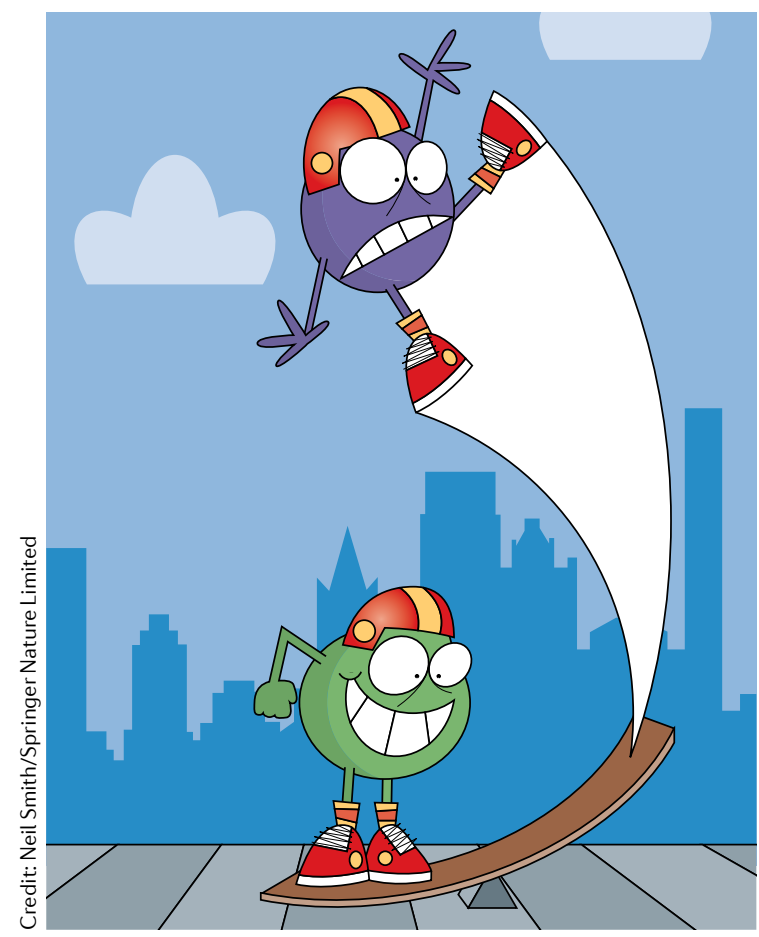

the level seen in APOE2 mice. Were $A P O E$ variants also affecting response to immunotherapy?

Looking into this question, the authors found that APOE4 mice bearing YUMMER1.7 tumours (a more immunogenic derivative of the YUMM1.7 model) survived longer on anti-PD1 treatment than APOE2 mice. These results were reflected by an analysis of published data from patients with advanced melanoma who received anti-PD1 therapy following progression on anti-CTLA4 therapy. This analysis showed improved survival of patients who carried the APOE4 variant compared with those carrying the $A P O E 3$ variant and shorter survival of patients who carried the APOE2 variant compared with those carrying APOE3.

Of note, the protein product of APOE4 has been reported to show enhanced binding to the APOE receptor and/or results in enhanced APOE signalling. Thus, APOE activation might further benefit patients with melanoma carrying the APOE4 variant. To test this hypothesis in vivo, the authors decided to target liver $\mathrm{x}$ receptors (LXRs), which transcriptionally activate genes in lipid and cholesterol metabolism, including APOE. Using RGX-104 as an LXR agonist, the authors found that YUMM1.7 tumours in APOE4 mice receiving RGX-104 grew significantly slower than in untreated APOE4 mice, whereas tumours in $A P O E 2$ mice did not show a change in growth in response to this treatment. This lack of response could be related to the reduced binding ability of APOE2 to APOE receptors, which cannot be rescued by LXR agonism.

Despite requiring validation in other models, this study offers the potential to identify patients with high risk for melanoma-associated death based on their $A P O E$ carrier status. $A P O E$ carrier status also has the potential to serve as a biomarker to identify patients who might benefit from immune checkpoint blockade and/or LXR agonism.

Ulrike Harjes

ORIGINAL ARTICLE Ostendorf, B. N. et al. Common germline variants of the human APOE gene modulate melanoma progression and survival. Nat.Med. https://doi.org/10.1038/ 441591-020-0879-3 (2020) 